\title{
Spin depolarizing effect in collisions of simple/complex atoms in spherically symmetric states with neutral hydrogen
}

\author{
M. Derouich ${ }^{1}$, P. S. Barklem ${ }^{2}$, and S. Sahal-Bréchot ${ }^{1}$ \\ 1 Observatoire de Paris-Meudon, LERMA UMR CNRS 8112, 5 place Jules Janssen, 92195 Meudon Cedex, France \\ e-mail: Moncef.Derouich@obspm. fr \\ 2 Department of Astronomy and Space Physics, Uppsala University, Box 515, 75120 Uppsala, Sweden
}

Received 18 March 2005 / Accepted 9 May 2005

\begin{abstract}
We present a general semi-classical treatment of the depolarization of spherically symmetric states (i.e. $s$-states) which necessarily accounts for the exchange interaction via exchange perturbation theory. Calculations of the destruction rate of orientation of general $s$-states, characterized by their effective principal quantum number $n^{*}$, due to isotropic collisions with neutral hydrogen have been carried out. It is found that the behaviour of the depolarization rates with $n^{*}$ obeys a power law. We express the depolarization and polarization transfer rates of complex atoms in terms of the depolarization rates of simple atoms. These results are used to infer the all non-zero depolarization and polarization transfer rates of the lower levels of the multiplets 42 and 145 of neutral Ti I. Further, we explain how our results can be used to easily calculate the hyperfine depolarization and polarization transfer rates. In order to validate our general theory, we have computed the destruction rate of orientation of the ground levels of the alkali metals Li I, Na I, K I, Rb I and Cs I using the quantum chemistry potentials of Geum et al. (2001, J. Ch. Phys., 115, 5984), employing our semi-classical description of the collision dynamics. For Na I ground state, the percentage error on our destruction rate of the orientation with respect to the fully quantal rate of Kerkeni et al. (2000b, A\&A, 364, 937; note Erratum) is less than $1 \%$ at $T=5000 \mathrm{~K}$.
\end{abstract}

Key words. Sun: atmosphere - atomic processes - line: formation

\section{Introduction}

Over the last few years, we have developed a general semiclassical theory of depolarization of spectral lines by collisions of levels of emitting/absorbing atoms/ions with neutral hydrogen (Derouich et al. 2003a,b, 2004a,b, 2005, hereafter Papers I, II, III, IV and V respectively). Our semi-classical theory is not specific to a given atom/ion and it is possible to apply the theory even to heavy atoms $(\mathrm{Ti}, \mathrm{Fe}, \ldots)$, which are very difficult or even impossible to treat with quantum chemistry methods, particularly in cases of excited states. Our method permits the calculation of the depolarization and polarization transfer rates for $p(l=1), d(l=2)$ and $f(l=3)$ levels of simple and complex atoms/ions. In our approach, spin is neglected since depolarization arising from collision induced transitions between Zeeman sublevels $M_{J} \rightarrow M_{J^{\prime}}$ is predominantly caused by collision induced transitions between Zeeman states with different projections of orbital angular momentum $\left(m_{l}\right)$.

In this paper we provide a general semi-classical treatment which accounts for spin to calculate s-states $(l=0)$ depolarizing rates. We use exchange perturbation theory, particularly that of Murrell \& Shaw (1967) and Musher \& Amos (1967), often referred to as MSMA theory, to evaluate the hydrogen-atom interaction potential. The interaction potential can be split into direct terms, corresponding to standard Rayleigh-Schrödinger perturbation theory, and exchange terms resulting from the consideration of electron exchange between the hydrogen atom and the perturbed atom/ion. Following Anstee (1992), we retain the important terms up to second order, and apply the Unsöld approximation (Unsöld 1927) where is assumed that the energy denominator in the second order terms of the perturbation can be replaced by a suitable average energy $E_{\mathrm{p}}$. As suggested by Anstee (1992), we adopt different values of $E_{\mathrm{p}}$ for the direct and exchange terms. We refer to the potentials calculated in this manner as Murrell-Shaw-Musher-Amos-Unsöld or MSMAU potentials. For our case of interest, a ground state hydrogen atom interacting with an atom in an s-state, this leads to singlet and triplet molecular states with interaction energies $V^{1} \Sigma$ and $V^{3} \Sigma$. These potentials enter standard semi-classical coupled differential equations describing the time evolution of the collision; the integration of these equations over an entire collision providing the scattering matrix pertaining to the $s(l=0)$ state, which may be used to calculate the depolarization rates of interest.

Our calculations show that the depolarization rates have a power law behaviour with effective principal quantum number $n^{*}$. We have also calculated the depolarization rates of s-states of alkali-atoms using accurate potential energy 
curves from Geum et al. (2001) ${ }^{1}$ computed by ab initio quantum chemistry methods. We compare the rates from the general treatement employing perturbation theory potentials with those obtained using the ab initio quantum chemistry potentials. We also compare our results for the Na I case with the results of Kerkeni et al. (2000b) where quantum chemistry potentials and a fully quantal description of the dynamics have been employed. Very satisfactory agreement is obtained.

This paper is a continuation of a series concerned with theoretical calculations of the collisional depolarization and polarization transfer rates. With the addition of this work depolarization rates may now be obtained with relative ease for $s(l=0), p(l=1), d(l=2)$ and $f(l=3)$ levels, making it possible to rapidly obtain the large number of depolarization rates for levels associated to lines of interest necessary for interpretation of the second solar spectrum.

\section{Formulation of the problem}

Our model for the system follows that described in preceding papers (see Paper I), now with the inclusion of spin. For sstates, the orbital angular momentum of the valence electron of the perturbed atom is $l=0$. If spin is neglected and the calculations performed in the $\left|n l m_{l}\right\rangle$ basis, transforming to the basis including spin $\left|n J M_{J}\right\rangle$ noting $J=s(s=1 / 2)$, and $M_{J}=M_{s}$, we have (Paper I, Eq. (20)):

$$
\begin{aligned}
\left\langle n_{1} s=\right. & \left.\frac{1}{2} M_{s}|S| n_{1} s=\frac{1}{2} M_{s}^{\prime}\right\rangle=2 \times \delta_{M_{s}, M_{s}^{\prime}} \\
& \times\left\langle n_{1} l=0 m_{l}=0|S| n_{1} l=0 m_{l}=0\right\rangle,
\end{aligned}
$$

where $\delta$ is the Kronecker symbol. In the case of spherically symmetric levels $J=1 / 2$, due to the fact that there is only a single $m_{l}=0$ level the only mechanism for collisional depolarization is via the spin exchange transition $M_{J}= \pm 1 / 2 \rightarrow M_{J}^{\prime}=$ $\mp 1 / 2$, and thus the neglect of the spin leads to null off-diagonal elements of the scattering matrix resulting in a zero destruction rate of the orientation of spherically symmetric $J=1 / 2$ levels. The spin flip transition may lead to destruction of orientation, but since a $J=1 / 2$ level is linearly unpolarised by definition not to destruction of alignment. Though the destruction rate of orientation is related to the circular atomic polarization, and thus not obviously relevant to the astrophysical interpretation of linearly polarization (see Paper I), it is necessary to calculate because:

1. as we will demonstrate in detail in Sect. 7, the depolarization and population transfer rates for some states of complex atoms may be expressed in terms of the destruction of orientation rates for simple atoms;

2. the hyperfine components into which a $J=1 / 2$ level splits in the coupling process with the nuclear spin (if it exists), may show linear polarization: the depolarization rates of hyperfine levels have to be calculated in order to interpret this linear polarization. Under typical conditions of formation of lines of the second solar spectrum, the frequency

\footnotetext{
${ }^{1}$ Tables of the ab initio potential curves of $\mathrm{LiH}, \mathrm{NaH}, \mathrm{KH}, \mathrm{RbH}$, and CsH Geum et al. (2001) are available in electronic format from the EPAPS homepage, cited as EPAPS in the references.
}

separations between the hyperfine levels of the $J=1 / 2$ level are very small compared to the inverse duration of a collision and the hyperfine splitting can be ignored in the treatment of the collision. Therefore, the off-diagonal scattering matrix elements of the hyperfine components are proportional to the off-diagonal scattering matrix between sublevels of the spherically symmetric $J=1 / 2$ level (Paper I, Eq. (19)) and, in the irreducible tensorial operator basis, the depolarization rates of the hyperfine levels are proportional to the destruction rate of the orientation of the spherically symmetric $J=1 / 2$ state (Nienhuis 1976; Omont 1977)

For the purpose of calculations of the depolarization rates mentioned in the points 1 . and 2., we must remove the neglect of spin in the calculation of the interaction potential and in the dynamics of the collision.

\section{Perturbation theory potentials including exchange}

In our previous works, the adiabatic potential curves were computed using conventional Rayleigh-Schödinger (RS) perturbation theory to second order, employing the Unsöld approximation (RSU) to remove the summation over all states in the second order term. The electron spins, and therefore the requirement that the electronic wavefunction of the system be antisymmetric with respect to exchange of the electrons, were neglected and thus the so-called exchange interactions were not considered.

To extend our theory to include the effects of spin we must use an exchange, sometimes called "symmetryadapted", perturbation theory. Many different formalisms exist for perturbation theory involving exchange interactions, some overviews are given by, for example, Chipman et al. (1973) or Hirschfelder (1967a,b). In this work, we will refer to the exchange perturbation theory of Murrell \& Shaw (1967) and Musher \& Amos (1967), hereafter MSMA theory. Different exchange perturbation theories generally differ in the details of the expressions for the second and higher order energies. It is worth noting that as we will describe below, we keep only the dominant terms at long range of the second order energy, and in fact these same terms would appear in second order also in other formalisms.

We apply the MSMA theory to consider the interaction of an atom $(\mathrm{A})$ with hydrogen atom $(\mathrm{H})$, following the model of Paper I with one electron associated to each atom. Their valence electrons are labelled by 1 and 2 respectively (Fig. 1). The system Hamiltonian is the sum of the atomic Hamiltonians and an interaction $V, H=H_{\mathrm{A}}+H_{\mathrm{H}}+V=$ $H_{0}+V . H_{\mathrm{A}}$ and $H_{\mathrm{H}}$ have electronic wavefunctions which satisfy $H_{\mathrm{A}}\left|J_{1} M_{J_{1}, i}\right\rangle=E_{i}\left|J_{1} M_{J_{1}, i}\right\rangle$ and $H_{\mathrm{H}}\left|J_{2} M_{J_{2}, j}\right\rangle=E_{j}$ $\left|J_{2} M_{J_{2}, j}\right\rangle$. Hereafter we denote by $J_{1}$ the total angular momentum of the perturbed atom and by $J_{2}$ that of the neutral hydrogen atom. The product functions $\left|J_{1} M_{J_{1}, i} J_{2} M_{J_{2}, j}\right\rangle$ satisfy $H_{0}\left|J_{1} M_{J_{1}, i} J_{2} M_{J_{2}, j}\right\rangle=\left(E_{i}+E_{j}\right)\left|J_{1} M_{J_{1}, i} J_{2} M_{J_{2}, j}\right\rangle$. $\left|J_{2} M_{J_{2}, j} J_{1} M_{J_{1}, i}\right\rangle$ is the product function where electron labels have been exchanged. To simplify the notation in the following, 


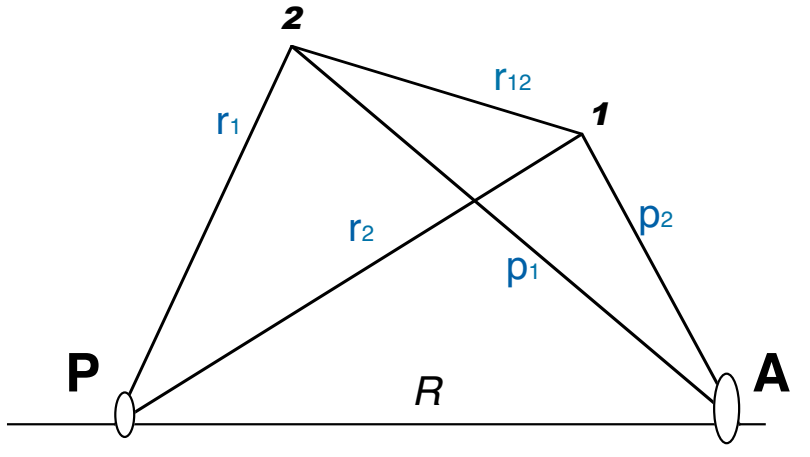

Fig. 1. The perturbed atom core is located at A, and the hydrogen perturbing core (a proton) at $\mathrm{P}$. Their valence electrons are denoted by 1 and 2 respectively. It is possible to produce the exchange term if the valence electrons are substituted.

we define $\left|a_{i}\right\rangle=\left|J_{1} M_{J_{1}, i}\right\rangle,\left|b_{j}\right\rangle=\left|J_{2} M_{J_{2}, j}\right\rangle$ and $E_{i j}=E_{i}+E_{j}$. For the unperturbed system in the state $\left|a_{0} b_{0}\right\rangle$, the first order correction to the energy due to the perturbation $V$ is, e.g. Murrell \& Shaw Eq. (37),

$E^{(1)}=\frac{\left\langle a_{0} b_{0}|V| a_{0} b_{0}\right\rangle \pm\left\langle a_{0} b_{0}|V| b_{0} a_{0}\right\rangle}{1 \pm S_{00}}$,

where $S_{00}=\left\langle a_{0} b_{0} \mid b_{0} a_{0}\right\rangle=\left\langle a_{0} \mid b_{0}\right\rangle^{2}$ is the wavefunction overlap. Note this is the Heitler-London energy. The second order term is more complicated, and may be derived in full from Eq. (48) of Murrell \& Shaw. However, the dominant term for the large to intermediate separations of interest here where overlap is small (that is, terms of order of the first order correction have been omitted) is

$E^{(2)} \approx \frac{1}{1 \pm S_{00}}$

$\times \sum_{i j}^{\prime} \frac{\left\langle a_{0} b_{0}|V| a_{i} b_{j}\right\rangle\left\langle a_{i} b_{j}|V| a_{0} b_{0}\right\rangle \pm\left\langle a_{0} b_{0}|V| a_{i} b_{j}\right\rangle\left\langle a_{i} b_{j}|V| b_{0} a_{0}\right\rangle}{E_{00}-E_{i j}}$

where the prime indicates the exclusion of the state $\left|a_{0} b_{0}\right\rangle$ from the summation. The singlet and triplet potentials, $V^{1} \Sigma$ and $V^{3 \Sigma}$ respectively, to second order are given by $E^{(1)}+E^{(2)}$ where the upper signs $(+)$ correspond to the singlet case and lower signs (-) the triplet case.

In both the first and second order expressions the first term is referred to as the direct term and the second the exchange term. As in previous work, we wish to simplify the second order calculation using the Unsöld approximation to remove the summation over all states. This is done by assuming an average energy denominator $E_{\mathrm{p}}(R)$, which allows completeness of the basis set to be used to remove the sum. Anstee (1992) has suggested the use of different values of $E_{\mathrm{p}}$ for the direct and exchange terms, since different states contribute more strongly in each case. That is,

$$
\begin{aligned}
E^{(2)} & \approx \frac{1}{E_{\mathrm{p}}(\text { dir })} \frac{\left\langle a_{0} b_{0}\left|V^{2}\right| a_{0} b_{0}\right\rangle}{1 \pm S_{00}} \\
& \pm \frac{1}{E_{\mathrm{p}}(\text { exch })} \frac{\left\langle a_{0} b_{0}\left|V^{2}\right| b_{0} a_{0}\right\rangle}{1 \pm S_{00}} .
\end{aligned}
$$

For our case of interest where one atom is a ground state hydrogen atom, and the other a generic neutral metal atom as described in Paper I, as in previous work we follow Unsöld (1955) and adopt $E_{\mathrm{p}}$ (dir) $=-4 / 9$ atomic units at all separations. It is less clear how one may choose a reasonable value for $E_{\mathrm{p}}(\mathrm{exch})$. Comparison of the matrix elements in Eq. (3), at least for low lying states, indicates that the states contributing strongly to the sum in the exchange term will generally be those with more extended wavefunctions and thus higher energies than those contributing to the direct term. Thus, we expect $E_{\mathrm{p}}($ exch $)<E_{\mathrm{p}}($ dir $)=-4 / 9$. In fact, the major contribution to the exchange integral sum is likely to come from continuum states, as may well be expected (see Musher \& Amos 1967). We will return to the choice of $E_{\mathrm{p}}(\mathrm{exch})$ below.

The model system and interaction $V$ is as defined in Paper I. The direct matrix elements have been computed as described in Anstee \& O’Mara (1991), namely integrations are performed analytically except for a final numerical integration over the radial wavefunction of the perturbed atom. The exchange matrix elements were computed following Anstee (1992) which should be consulted for details. The technique is similar to that for direct matrix elements; however, due to the exchange of electron labels, two integrals must be performed numerically and terms containing $1 / r_{12}$ are only tractable analytically if expressed as expansions in terms of Legendre polynomials as well as containing nested integrals leading to some numerical difficulties. The calculations were performed using code written by Stuart Anstee, detailed in Anstee (1992), where we have made improvements to the numerical properties.

It still remains to set $E_{\mathrm{p}}$ (exch). To try and obtain some guidance, we have investigated the $E_{\mathrm{p}}(\mathrm{exch})$ values required to achieve reasonable agreement with the quantum chemistry potentials of Geum et al. (2001) for the $\mathrm{LiH}, \mathrm{NaH}, \mathrm{KH}, \mathrm{RbH}$ and $\mathrm{CsH}$ ground states. Such systems are very similar to our model, namely, a single valence electron outside a more tightly bound, spherically symmetric core, and thus $E_{\mathrm{p}}(\mathrm{exch})$ inferred from these systems will be appropriate for our model, at least at intermediate to long range where the core has little influence. In making any comparisons, one must keep in mind that the singlet states of the quantum chemistry potentials have avoided ionic crossings in the region $R \sim 6-10 a_{0}$ and thus are strongly ionic in character at around $R \leq 12 a_{0}$. The ionic configuration is not included in our calculations; we are interested in longer range interactions. It should be noted anyway that at short range perturbation theory may breakdown in general, or other orders may become important. We note that the coupling between different spin states in the dynamic equations (see Sect. 4, Eq. (7)), which leads to off-diagonal scattering matrix elements and thus depolarization, depends on the splitting $V^{3} \Sigma-V^{1} \Sigma$, which in the limit of small overlap is determined by the exchange terms. We computed MSMAU potentials for each case and optimised $E_{\mathrm{p}}(\mathrm{exch})$ so that the exchange splitting $V^{3} \Sigma-V^{1 \Sigma}$ best matched that of the quantum chemistry potential in the region $R \sim 15-25 a_{0}$. In some cases the comparisons were restricted to separations less than $25 a_{0}$ as it was clear that the numerical precision of the quantum chemistry potentials was insufficient to avoid roundoff error. For the cases of the $\mathrm{LiH}$, $\mathrm{NaH}, \mathrm{KH}, \mathrm{RbH}$ and $\mathrm{CsH}$ ground states we found $E_{\mathrm{p}}(\mathrm{exch}) \sim$ $-7.6,-5.4,-3.8,-2.2,-1.2$ best reproduced the splitting of the triplet and singlet electronic states in this region. As might 
be expected, $\left|E_{\mathrm{p}}(\mathrm{exch})\right|$ correlates with binding energy of the perturbed atom state, having respectively effective principal quantum numbers $n^{*}=1.588,1.627,1.770,1.804,1.869$, recalling $n^{*}=\left[2 E_{\infty}\right]^{-1 / 2}, E_{\infty}$ being the binding energy of the state in atomic units. Such a correlation is expected since as $n^{*}$ increases the electronic wavefunction becomes more extended, and thus an expansion in states centred on the hydrogen atom nucleus, would have larger contributions from continuum states with larger wavelengths and thus lower energies. As discussed above, on physical grounds at the separations of interest $E_{\mathrm{p}}(\mathrm{exch})$ should not exceed $E_{\mathrm{p}}$ (dir). It is possible to make a good fit to the above inferred values for $E_{\mathrm{p}}(\mathrm{exch})$ and account for this constraint by assuming an exponential dependence of $E_{\mathrm{p}}(\mathrm{exch})$ on $n^{*}$, specifically

$E_{\mathrm{p}}(\mathrm{exch})=-0.4444-\exp \left(-7.017 n^{*}+13.157\right)$.

The usefulness of this estimate will naturally be determined by the sensitivity of the depolarization rate to this parameter over a reasonable range, which will be investigated below (Sect. 6, Fig. 3). A comparison of our potentials with the quantum chemistry potentials is shown in Fig. 2 for the case of $\mathrm{NaH}$.

\section{Dynamics of the collision}

Let us consider collisions between an atom (A) with a ground configuration $n \mathrm{~s}\left({ }^{2} \mathrm{~S}_{1 / 2}\right)$ and a hydrogen atom $(\mathrm{H})$ in its ground state $1 \mathrm{~s}\left({ }^{2} \mathrm{~S}_{1 / 2}\right)$. The molecular states correlated to the $\mathrm{A}\left({ }^{2} \mathrm{~S}\right)+\mathrm{H}\left({ }^{2} \mathrm{~S}\right)$ asymptote are the attractive ${ }^{1} \Sigma$ state and the repulsive ${ }^{3} \Sigma$ state. We assume that for the regions of interest the wavefunction overlap is negligible, and so the perturbed electronic wavefunction of the system may be expanded over the product states of the configuration:

$$
\begin{aligned}
|\psi(t)\rangle= & a_{1}(t)\left|J_{1} \frac{1}{2}\right\rangle\left|J_{2} \frac{1}{2}\right\rangle \mathrm{e}^{-\mathrm{i} E_{1}^{0} t} \\
& +a_{1}(t)\left|J_{1} \frac{-1}{2}\right\rangle\left|J_{2} \frac{-1}{2}\right\rangle \mathrm{e}^{-\mathrm{i} E_{2}^{0} t} \\
& +a_{3}(t)\left|J_{1} \frac{1}{2}\right\rangle\left|J_{2} \frac{-1}{2}\right\rangle \mathrm{e}^{-\mathrm{i} E_{3}^{0} t} \\
& +a_{4}(t)\left|J_{1} \frac{-1}{2}\right\rangle\left|J_{2} \frac{1}{2}\right\rangle \mathrm{e}^{-\mathrm{i} E_{4}^{0} t}
\end{aligned}
$$

where $\left|J_{1} M_{J_{1}}\right\rangle\left|J_{2} M_{J_{2}}\right\rangle$ are the eigenfunctions of the unperturbed system corresponding to the eigenvalues $E_{i}^{0}$. The coupled differential equations, derived from the time-dependent Schrödinger equation, are in the fixed laboratory frame (Roueff 1974):

$\mathrm{i} \frac{\partial a_{1}(t)}{\partial t}=a_{1}(t) V^{3} \Sigma$

$\mathrm{i} \frac{\partial a_{2}(t)}{\partial t}=a_{2}(t) V^{3 \Sigma}$

$\mathrm{i} \frac{\partial a_{3}(t)}{\partial t}=\frac{1}{2}\left(V^{3} \Sigma+V^{1 \Sigma}\right) a_{3}(t)+\frac{1}{2}\left(V^{3 \Sigma}-V^{1 \Sigma}\right) a_{4}(t)$

$\mathrm{i} \frac{\partial a_{4}(t)}{\partial t}=\frac{1}{2}\left(V^{3} \Sigma-V^{1} \Sigma\right) a_{3}(t)+\frac{1}{2}\left(V^{3} \Sigma+V^{1} \Sigma\right) a_{4}(t)$

where $V^{1} \Sigma$ and $V^{3} \Sigma$ are respectively the energy of the singlet and triplet molecular states with zero angular momentum along the
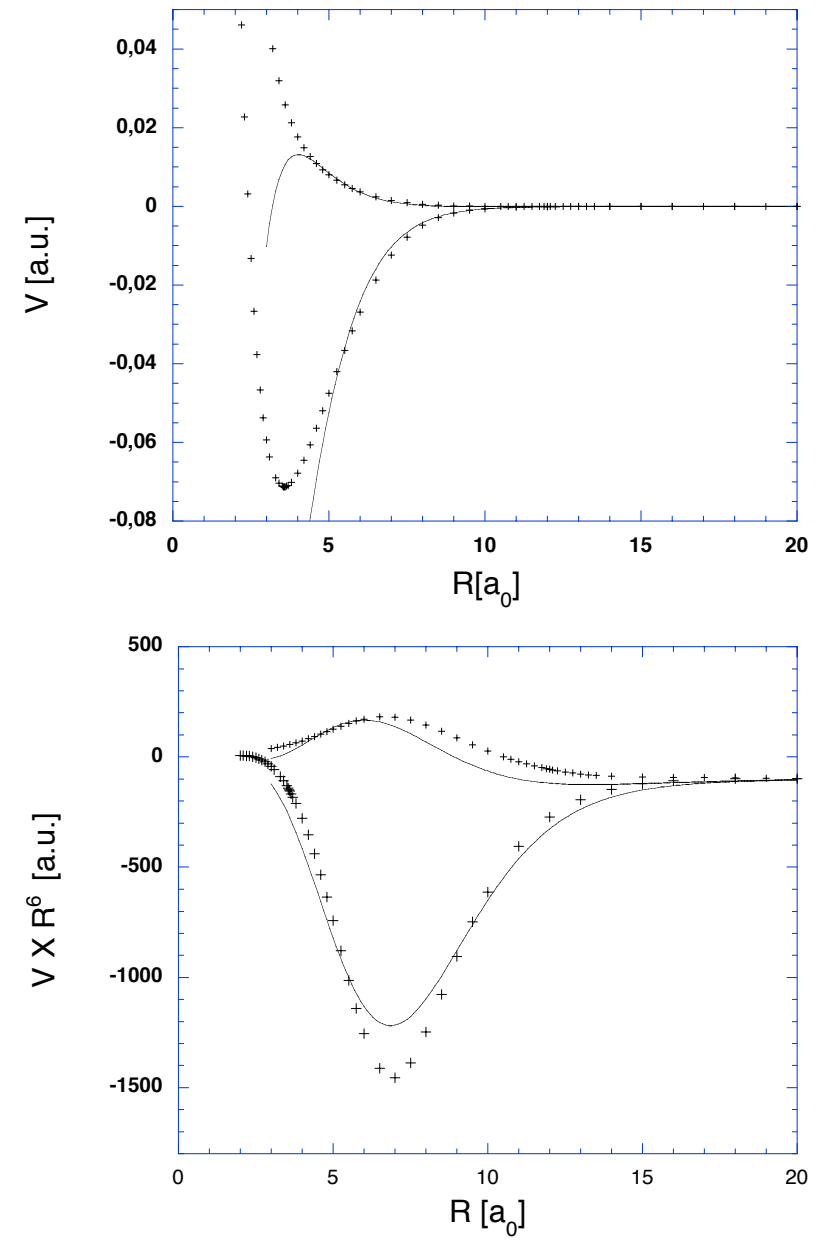

Fig. 2. Comparison of adiabatic potential curves for the ground state of NaH. The MSMAU potentials computed in this work are plotted as full lines, the quantum chemistry potentials of Geum et al. (2001) as crosses. Note that for the MSMAU potentials $E_{\mathrm{p}}$ (exch) has been chosen to match the splitting of the Geum et al. potentials at 15-20 $a_{0}$. The upper plot shows the potentials $V^{3 \Sigma}$ (upper curves) and $V^{1 \Sigma}$ (lower curves); the lower plot shows the same potentials scaled by $R^{6}$ to better display the behaviour at larger separations.

interatomic axis, as usual computed in the molecular (rotating) frame. The integration of these equations over an entire collsion $(t=-\infty \rightarrow t=+\infty)$ provides the 16 scattering matrix elements $\left\langle J_{1} M_{J_{1}}\left|\left\langle J_{2} M_{J_{2}}\left|S_{\mathrm{sc}}\right| J_{2} M_{J_{2}}^{\prime}\right\rangle\right| J_{1} M_{J_{1}}^{\prime}\right\rangle$.

Note, as mentioned earlier, for s-states the depolarization arises only from the transitions between states of different projections of spin. Due to the spherical symmetry the equations are not dependent on the angle between the laboratory and rotating frames. However, the semi-classical coupled linear differential equations for non-spherically symmetric $\mathrm{p}, \mathrm{d}$ and $f$ states depend on this angle and the depolarizing effect is caused by transitions between Zeeman sublevels with different $m_{l}$ (e.g., see Paper I).

\section{Semi-classical definition of the depolarization probability}

Since the collisions are isotropic, in order to calculate the depolarization probability we must average over all possible 


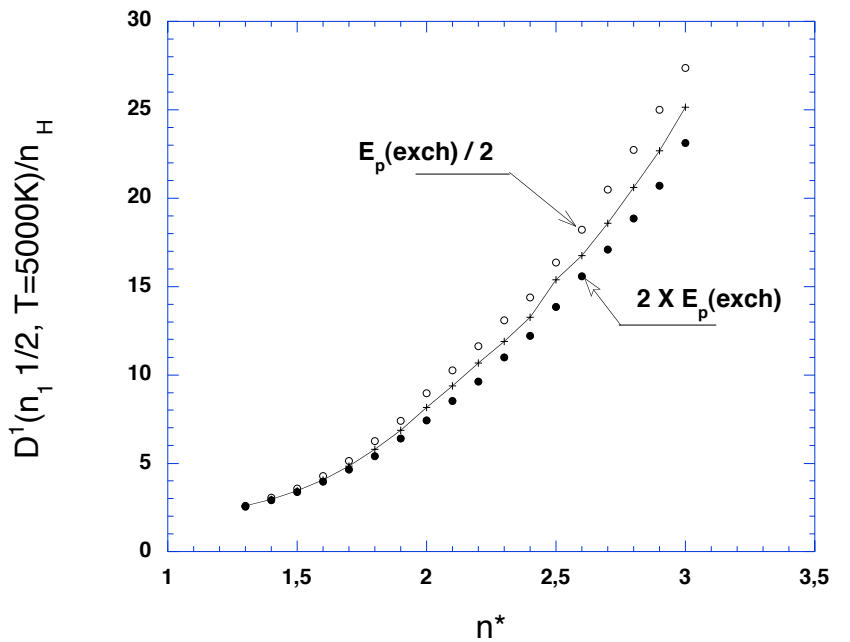

Fig. 3. Dependence on $E_{\mathrm{p}}$ (exch) of the depolarization rates $D^{1}\left(n_{1} 1 / 2,5000 \mathrm{~K}\right) / n_{\mathrm{H}}$ of s-states for different values of $n^{*}$. Full line crosses: results with the best $E_{\mathrm{p}}(\mathrm{exch})$; filled circles: results for $2 \times$ $E_{\mathrm{p}}(\mathrm{exch})$; open circles: results for $E_{\mathrm{p}}(\mathrm{exch}) / 2$. The rates are given in $10^{-15} \mathrm{rad} \mathrm{m}^{3} \mathrm{~s}^{-1}$.

orientations of the collision plane $(\boldsymbol{b}, \boldsymbol{v})$. The only non-zero depolarization probability is that of rank $k=1$ when expressed in the irreducible tensorial operator basis formed by the eigenvectors ${ }^{n_{1} J_{1}} T_{0}^{k} \otimes^{n_{2} J_{2}} T_{0}^{0}$ where

$$
\begin{aligned}
{ }^{n_{1} J_{1}} T_{0}^{k}= & (2 k+1) \times \sum_{M_{J_{1}} M_{J_{1}}^{\prime}}(-1)^{J_{1}-M_{J_{1}}} \\
& \times\left(\begin{array}{ccc}
J_{1} & k & J_{1} \\
-M_{J_{1}} & 0 & M_{J_{1}}^{\prime}
\end{array}\right)\left|n_{1} J_{1} M_{J}\right\rangle\left\langle n_{1} J_{1} M_{J}^{\prime}\right|,
\end{aligned}
$$

and

${ }^{n_{2} J_{2}} T_{0}^{0}=\frac{1}{\sqrt{2}}\left(\left|n_{2} J_{2} \frac{1}{2}\right\rangle\left\langle n_{2} J_{2} \frac{1}{2}|+| n_{2} J_{2} \frac{-1}{2}\right\rangle\left\langle n_{2} J_{2} \frac{-1}{2}\right|\right)$.

In the irreducible tensorial operator basis, using properties of the rotation matrices (Messiah 1961) and after some angular algebra transformations, we find that the angular average of the depolarization probability is given by:

$$
\begin{aligned}
& \left\langle P^{k}\left(n_{1} J_{1}, b, v\right)\right\rangle_{\mathrm{av}}=\frac{1}{\left(2 J_{1}+1\right)} \sum_{\gamma_{2}, \gamma_{2}^{\prime}} \frac{1}{\left(2 J_{2}+1\right)} \\
& \times \sum_{\mu_{1}, \mu_{1}^{\prime}}\left|\left\langle J_{1} \mu_{1}\left|\left\langle J_{2} \gamma_{2}|T(\boldsymbol{b}, \boldsymbol{v})| J_{2} \gamma_{2}^{\prime}\right\rangle\right| J_{1} \mu_{1}^{\prime}\right\rangle\right|^{2} \\
& -\sum_{\gamma_{2}, \gamma_{2}^{\prime}, \beta_{2}, \beta_{2}^{\prime}} \sum_{\mu_{1}, \mu_{1}^{\prime}, v_{1}, v_{1}^{\prime}}\left\langle J_{1} \mu_{1}\left|\left\langle J_{2} \gamma_{2}|T(\boldsymbol{b}, \boldsymbol{v})| J_{2} \gamma_{2}^{\prime}\right\rangle\right| J_{1} \mu_{1}^{\prime}\right\rangle \\
& \times\left\langle J_{1} v_{1}\left|\left\langle J_{2} \beta_{2}|T(\boldsymbol{b}, \boldsymbol{v})| J_{2} \beta_{2}^{\prime}\right\rangle\right| J_{1} v_{1}^{\prime}\right\rangle^{*} \\
& \times \sum_{\alpha_{1}}(-1)^{2 J_{1}+k+\mu_{1}-\mu_{1}^{\prime}}\left(\begin{array}{ccc}
J_{1} & J_{1} & k \\
-v_{1}^{\prime} \mu_{1}^{\prime} & \alpha_{1}
\end{array}\right)\left(\begin{array}{ccc}
J_{1} & J_{1} & k \\
v_{1}-\mu_{1}-\alpha_{1}
\end{array}\right) \\
& \times \sum_{\alpha_{2}}(-1)^{2 J_{2}+k_{2}+\beta_{2}-\beta_{2}^{\prime}}\left(\begin{array}{cc}
J_{2} & J_{2} k_{2} \\
-\gamma_{2}^{\prime} \beta_{2}^{\prime} \alpha_{2}
\end{array}\right)\left(\begin{array}{ccc}
J_{2} & J_{2} & k_{2} \\
\gamma_{2} & -\beta_{2} & -\alpha_{2}
\end{array}\right) \text {. }
\end{aligned}
$$

Taking $k_{2}=0$ since the collisional depolarization concerns only the states of the perturbed atom $\left|n_{1} J_{1} \mu_{1}\right\rangle$, Eq. (10) becomes:

$$
\begin{aligned}
& \left\langle P^{k}\left(n_{1} J_{1}, b, v\right)\right\rangle_{\mathrm{av}}=\frac{1}{\left(2 J_{1}+1\right)} \sum_{\mu_{1}, \mu_{1}^{\prime}} \frac{1}{\left(2 J_{2}+1\right)} \sum_{\gamma_{2}, \gamma_{2}^{\prime}} \\
& \times\left|\left\langle J_{1} \mu_{1}\left|\left\langle J_{2} \gamma_{2}|T(\boldsymbol{b}, \boldsymbol{v})| J_{2} \gamma_{2}^{\prime}\right\rangle\right| J_{1} \mu_{1}^{\prime}\right\rangle\right|^{2} \\
& -\sum_{\mu_{1}, \mu_{1}^{\prime}, v_{1}, v_{1}^{\prime}} \sum_{\gamma_{2}, \gamma_{2}^{\prime}} \frac{1}{2 J_{2}+1}\left\langle J_{1} \mu_{1}\left|\left\langle J_{2} \gamma_{2}|T(\boldsymbol{b}, \boldsymbol{v})| J_{2} \gamma_{2}^{\prime}\right\rangle\right| J_{1} \mu_{1}^{\prime}\right\rangle \\
& \times\left\langle J_{1} v_{1}\left|\left\langle J_{2} \beta_{2}|T(\boldsymbol{b}, \boldsymbol{v})| J_{2} \beta_{2}^{\prime}\right\rangle\right| J_{1} v_{1}^{\prime}\right\rangle^{*} \\
& \times \sum_{\alpha_{1}}(-1)^{2 J_{1}+k+\mu_{1}-\mu_{1}^{\prime}}\left(\begin{array}{ccc}
J_{1} & J_{1} & k \\
-v_{1}^{\prime} \mu_{1}^{\prime} & \alpha_{1}
\end{array}\right)\left(\begin{array}{ccc}
J_{1} & J_{1} & k \\
v_{1}-\mu_{1}-\alpha_{1}
\end{array}\right) \text {. }
\end{aligned}
$$

Note, $T(\boldsymbol{b}, \boldsymbol{v})=I-S_{\mathrm{sc}}(\boldsymbol{b}, \boldsymbol{v})(I$ is the unit matrix $)$ is the so-called transition matrix computed in the atomic frame (equivalent to the laboratory frame of earlier) in which the perturbed atom is stationary at the origin, and the quantization axis $(z)$ is taken to be perpendicular to the collision plane $(\boldsymbol{b}, \boldsymbol{v})$. Equation (11) above generalizes those of Gordeyev et al. (1969) and MasnouSeeuws \& Roueff (1972) for a perturber with $J_{2}=0$ (He I). Note, when there is no fine structure transfer, which is the case for the $n \mathrm{~s}\left({ }^{2} \mathrm{~S}_{1 / 2}\right)$ state treated here, this definition of the depolarization probability is equivalent to that defined by Kerkeni et al. (2000a) Eq. (12). However, if there is fine structure transfer from atomic state $J_{1}^{\prime}$ to state $J_{1}$ (e.g. $4 \mathrm{p}^{2} \mathrm{P}_{3 / 2} \rightarrow 4 \mathrm{p}{ }^{2} \mathrm{P}_{1 / 2}$ ) our definition and the definition adopted by Kerkeni et al. are not the same because, unlike Kerkeni et al. (2002) Eq. (2), the term corresponding to the fine structure transfer between the levels is not included in Eq. (11).

The semi-classical depolarization rate $D^{k}\left(n_{1} J_{1}, T\right)$ follows from integration over impact parameters and velocities with a Maxwellian distribution $f(v, T)$ for a local temperature $T$ :

$$
\begin{aligned}
D^{k}\left(n_{1} J_{1}, T\right) \simeq & n_{\mathrm{H}} \int_{0}^{\infty} v f(v, T) \mathrm{d} v\left(\pi b_{0}^{2}\right. \\
& \left.+2 \pi \int_{b_{0}}^{\infty}\left\langle P^{k}\left(n_{1} J_{1}, b, v\right)\right\rangle_{\mathrm{av}} b \mathrm{~d} b\right)
\end{aligned}
$$

where $n_{\mathrm{H}}$ is the neutral hydrogen density in $\mathrm{m}^{3}$ and $b_{0}$ is the cutoff impact-parameter; we adopt $b_{0}=3 a_{0}$ as in previous work. One can verify easily via the definition of the depolarization probability given in Eq. (11) that destruction rates of population $D^{0}\left(n_{1} J_{1}, T\right)$ are zero. It is of interest to remark that $D^{k}\left(n_{1} J_{1}, T\right)$ is given by:

$$
D^{k}\left(n_{1} J_{1}, T\right)=\zeta^{0}\left(n_{1} J_{1}\right)-\zeta^{k}\left(n_{1} J_{1}\right)
$$

$\zeta^{k}\left(n_{1} J_{1}\right)$ is the spherical tensor component of the elastic rates due to isotropic collisions with hydrogen which is given by:

$$
\begin{aligned}
\zeta^{k}\left(n_{1} J_{1}, T\right) \simeq & n_{\mathrm{H}} \int_{0}^{\infty} v f(v, T) \mathrm{d} v\left(\pi b_{0}^{2}\right. \\
& \left.+2 \pi \int_{b_{0}}^{\infty}\left\langle Q^{k}\left(n_{1} J_{1}, b, v\right)\right\rangle_{\text {av }} b \mathrm{~d} b\right)
\end{aligned}
$$


where,

$$
\begin{aligned}
& \left\langle Q^{k}\left(n_{1} J_{1}, b, v\right)\right\rangle=\sum_{\mu_{1}, \mu_{1}^{\prime}, v_{1}, v_{1}^{\prime}} \sum_{\gamma_{2}, \gamma_{2}^{\prime}} \frac{1}{2 J_{2}+1} \\
& \times\left\langle J_{1} \mu_{1}\left|\left\langle J_{2} \gamma_{2}|T(\boldsymbol{b}, \boldsymbol{v})| J_{2} \gamma_{2}^{\prime}\right\rangle\right| J_{1} \mu_{1}^{\prime}\right\rangle \\
& \times\left\langle J_{1} v_{1}\left|\left\langle J_{2} \beta_{2}|T(\boldsymbol{b}, \boldsymbol{v})| J_{2} \beta_{2}^{\prime}\right\rangle\right| J_{1} v_{1}^{\prime}\right\rangle^{*} \\
& \times \sum_{\alpha_{1}}(-1)^{2 J_{1}+k+\mu_{1}-\mu_{1}^{\prime}}\left(\begin{array}{ccc}
J_{1} & J_{1} & k \\
-v_{1}^{\prime} & \mu_{1}^{\prime} & \alpha_{1}
\end{array}\right)\left(\begin{array}{ccc}
J_{1} & J_{1} & k \\
v_{1}-\mu_{1}-\alpha_{1}
\end{array}\right) \text {. }
\end{aligned}
$$

In addition $\zeta^{k}\left(n_{1} J_{1}, T\right)$ can be written as linear combination of the collisional transition rates in the dyadic basis $\left|J_{1} \mu_{1}\right\rangle\left\langle J_{1} \mu_{1}^{\prime}\right|$ :

$$
\begin{aligned}
\zeta^{k}\left(n_{1} J_{1}\right)= & (2 k+1) \sum_{\mu_{1}, \mu_{1}^{\prime}} \zeta\left(n_{1} J_{1} \mu_{1} \rightarrow n_{1} J_{1} \mu_{1}^{\prime}, T\right) \\
& \times(-1)^{\mu_{1}-\mu_{1}^{\prime}}\left(\begin{array}{ccc}
J_{1} & J_{1} & k \\
\mu_{1} & -\mu_{1} & 0
\end{array}\right)\left(\begin{array}{ccc}
J_{1} & J_{1} & k \\
\mu_{1}^{\prime} & -\mu_{1}^{\prime} & 0
\end{array}\right) .
\end{aligned}
$$

In particular,

$$
\begin{aligned}
\zeta^{0}\left(n_{1} J_{1}, T\right)= & \frac{1}{\left(2 J_{1}+1\right)} \sum_{\mu_{1}, \mu_{1}^{\prime}} \zeta\left(n_{1} J_{1} \mu_{1} \rightarrow n_{1} J_{1} \mu_{1}^{\prime}, T\right) \\
= & \frac{1}{\left(2 J_{1}+1\right)} \sum_{\mu_{1}, \mu_{1}^{\prime}} \frac{1}{\left(2 J_{2}+1\right)} \\
& \times \sum_{\gamma_{2}, \gamma_{2}^{\prime}} \zeta\left(n_{1} J_{1} \mu_{1} J_{2} \gamma_{2} \rightarrow n_{1} J_{1} \mu_{1}^{\prime} J_{2} \gamma_{2}^{\prime}, T\right)
\end{aligned}
$$

is the elastic collisional rate for the level $\left|n_{1} J\right\rangle$. Only the destruction rate of orientation $D^{1}\left(n \frac{1}{2}, T\right)=\zeta^{0}\left(n_{1} J_{1}, T\right)-$ $\zeta^{1}\left(n_{1} \quad J_{1}, T\right)$ is non-zero and can be written as a linear combination of the collisional transition rates between sublevels $\zeta\left(n_{1} \frac{1}{2} \mu_{1} \rightarrow n_{1} \frac{1}{2} \mu_{1}^{\prime}, T\right)$ :

$$
\begin{aligned}
D^{1}\left(n_{1} \frac{1}{2}, b, v\right)= & \zeta^{0}\left(n_{1} \frac{1}{2}\right)-3 \times \sum_{\mu_{1}, \mu_{1}^{\prime}}(-1)^{\mu_{1}-\mu_{1}^{\prime}} \\
& \times\left(\begin{array}{ccc}
\frac{1}{2} & \frac{1}{2} & 1 \\
\mu_{1} & -\mu_{1} & 0
\end{array}\right)\left(\begin{array}{ccc}
\frac{1}{2} & \frac{1}{2} & 1 \\
\mu_{1}^{\prime} & -\mu_{1}^{\prime} & 0
\end{array}\right) \\
& \times \zeta\left(n_{1} \frac{1}{2} \mu_{1} \rightarrow n_{1} \frac{1}{2} \mu_{1}^{\prime}\right) \\
= & \zeta\left(n_{1} \frac{1}{2} \frac{1}{2} \rightarrow n_{1} \frac{1}{2} \frac{-1}{2}\right) \\
& +\zeta\left(n_{1} \frac{1}{2} \frac{-1}{2} \rightarrow n_{1} \frac{1}{2} \frac{1}{2}\right) \\
= & 2 \times \zeta\left(n_{1} \frac{1}{2} \frac{1}{2} \rightarrow n_{1} \frac{1}{2} \frac{-1}{2}\right) .
\end{aligned}
$$

\section{Results}

\subsection{Results for spherically symmetric states}

In the MSMAU potential calculations $E_{\mathrm{p}}(\mathrm{exch})$ is adjusted empirically to best match the splitting of the Geum et al. potentials (see Sect. 3). As a check on the sensivity of the depolarization rates to the precision of the choice of $E_{\mathrm{p}}(\mathrm{exch})$, we have calculated the destruction rate of orientation $D^{1}\left(n_{1} 1 / 2,5000 \mathrm{~K}\right)$ varying $E_{\mathrm{p}}(\mathrm{exch})$ in Eq. (4); rates are calculated for the best
Table 1. Destruction of orientation rates for $T=5000 \mathrm{~K}$ and the velocity exponent as function of effective principal quantum number. Rates are given in $10^{-15} \mathrm{rad} \mathrm{m}^{3} \mathrm{~s}^{-1}$.

\begin{tabular}{lcc}
\hline \hline$n^{*}$ & $D^{1}\left(n_{1} \frac{1}{2}, 5000 \mathrm{~K}\right)$ & $\lambda^{1}\left(n_{1} \frac{1}{2}\right)$ \\
\hline 1.5 & 3.44 & 0.177 \\
1.6 & 4.06 & 0.177 \\
1.7 & 4.84 & 0.180 \\
1.8 & 5.78 & 0.174 \\
1.9 & 6.85 & 0.156 \\
2 & 8.156 & 0.168 \\
2.1 & 9.37 & 0.160 \\
2.2 & 10.67 & 0.155 \\
2.3 & 11.90 & 0.157 \\
2.4 & 13.27 & 0.165 \\
2.5 & 15.39 & 0.175 \\
2.6 & 16.75 & 0.161 \\
2.7 & 18.59 & 0.166 \\
2.8 & 20.60 & 0.158 \\
2.9 & 22.70 & 0.163 \\
3 & 25.15 & 0.171 \\
\hline
\end{tabular}

estimate of $E_{\mathrm{p}}(\mathrm{exch})$ given by Eq. (5) and for cases where we multiply or divide $E_{\mathrm{p}}(\mathrm{exch})$ by a factor of two. Figure 3 gives $D^{1}\left(n_{1} 1 / 2,5000 \mathrm{~K}\right)$ as function of $n^{*}\left(1.3 \leq n^{*} \leq 3\right.$.) for each value of $E_{\mathrm{p}}(\mathrm{exch})$. We remark that the rates show only weak variation with $E_{\mathrm{p}}(\mathrm{exch})$. For example for $n^{*}=1.6$, $D^{1}\left(n_{1} 1 / 2,5000 \mathrm{~K}\right)=4.06 \times 10^{-15} \mathrm{rad} \mathrm{m}^{3} \mathrm{~s}^{-1}$ for our best estimate of $E_{\mathrm{p}}(\mathrm{exch})$ and $3.96 \times 10^{-15} \mathrm{rad} \mathrm{m}^{3} \mathrm{~s}^{-1}$ if $E_{\mathrm{p}}(\mathrm{exch})$ is a factor of two larger: the difference is only $\sim 2.5 \%$. The sensitivity to the precision of $E_{\mathrm{p}}(\mathrm{exch})$ increases with $n^{*}$ (since $\left|E_{\mathrm{p}}(\mathrm{exch})\right|$ is smaller and the splitting is inversely proportional to $\left.\left|E_{\mathrm{p}}(\mathrm{exch})\right|\right)$ but even for $n^{*}=3$ the difference is only $8 \%$. Anyway, for ground states of alkali atoms $1.588 \leq n^{*} \leq 1.804$ and in general for s-states, the range of interest for interpretation of the second solar spectrum is $1.5 \leq n^{*} \leq 2$. For instance, $n^{*}=1.504$ and 1.636 for the lower levels of the multiplets 42 and 145 of Ti which treated in the present paper (next section).

As already seen in Papers I, II, III, IV, V, we found again that all the rates vary with temperature obeying a power law of the form $D^{1}\left(n_{1} 1 / 2, T=5000 \mathrm{~K}\right)\left(\frac{T}{5000}\right)^{\left(1-\lambda^{1}\left(n_{1} \frac{1}{2}\right)\right) / 2}$. Table 1 gives $D^{1}\left(n_{1} 1 / 2, T=5000 \mathrm{~K}\right)$ and the so-called velocity exponent $\lambda^{1}\left(n_{1} 1 / 2\right)$ as a function of effective principal quantum number $n^{*}$. The velocity exponent $\lambda^{1}\left(n_{1} 1 / 2\right)$ exhibits only a weak variation with $n^{*}$ and on average is 0.168 (i.e. $\left.\left(1-\lambda^{1}\left(n_{1} \frac{1}{2}\right)\right) / 2=0.416\right)$. However, $D^{1}\left(n_{1} 1 / 2, T=5000 \mathrm{~K}\right)$ has a striking power law behaviour with $n^{*}$ (see Fig. 4), which is fit by:

$D^{1}\left(n_{1} 1 / 2, T=5000 \mathrm{~K}\right)=1.0045 \times 10^{-15} n_{\mathrm{H}} \times n^{*^{2.979}}$.

In the other hand we have similarly calculated the spherical tensor component of the elastic collisional rates $\zeta^{0}\left(n_{1} 1 / 2, T\right)$ and $\zeta^{1}\left(n_{1} 1 / 2, T\right)$

$$
\begin{aligned}
\zeta^{0}\left(n_{1} 1 / 2, T=5000 \mathrm{~K}\right)= & {\left[31.214-53.630 \times n^{*}\right.} \\
& \left.+25.177 \times n^{*^{2}}\right] \times 10^{-15} n_{\mathrm{H}}
\end{aligned}
$$


and,

$$
\begin{aligned}
\zeta^{1}\left(n_{1} 1 / 2, T=5000 \mathrm{~K}\right)= & {\left[27.234-48.575 \times n^{*}\right.} \\
& \left.+21.88 \times n^{*^{2}}\right] \times 10^{-15} n_{\mathrm{H}} .
\end{aligned}
$$

As for the depolarization rate $D^{1}\left(n_{1} 1 / 2, T\right)$, we find that $\zeta^{0}\left(n_{1} 1 / 2, T\right)$ and $\zeta^{1}\left(n_{1} 1 / 2, T\right)$ can be written as a function of the local temperature $T$ where $(1-\lambda) / 2 \simeq 0.416$. These relationships will be used to calculate depolarization rates of complex atoms (Sect. 7) and may also be used to calculate hyperfine depolarization rates (Sect. 8).

\subsection{Results for alkali atoms in the ground state}

The effective principal quantum numbers of the Li I, Na I, K I, $\mathrm{Rb}$ I and Cs I ground states are $n^{*} \simeq 1.588,1.627,1.770,1.804$, and 1.869 respectively. The destruction rates of orientation for the ground states of alkali atoms can be either determined directly from the code associated to our theory or inferred from Eq. (19). We have used our code to compute directly the rates for $100 \leq T \leq 10000 \mathrm{~K}$ and we find the following analytical expressions in $\mathrm{rad} \mathrm{s}^{-1}$ :

$$
\begin{aligned}
& \text { - Li I }\left(2 \mathrm{~s}^{2} \mathrm{~S}_{1 / 2}\right)-\mathrm{HI}\left(1 \mathrm{~s}^{2} \mathrm{~S}_{1 / 2}\right) \text { : } \\
& D^{1}(211 / 2, T)=3.99 \times 10^{-15} \times n_{\mathrm{H}}\left(\frac{T}{5000}\right)^{0.410} \\
& \text { - NaI }\left(3 \mathrm{~s}^{2} \mathrm{~S}_{1 / 2}\right)-\mathrm{HI}\left(1 \mathrm{~s}^{2} \mathrm{~S}_{1 / 2}\right) \\
& D^{1}(311 / 2, T)=4.28 \times 10^{-15} \times n_{\mathrm{H}}\left(\frac{T}{5000}\right)^{0.408} \\
& \text { - KI }\left(4 \mathrm{~s}^{2} \mathrm{~S}_{1 / 2}\right)-\mathrm{HI}\left(1 \mathrm{~s}^{2} \mathrm{~S}_{1 / 2}\right) \text { : } \\
& D^{1}(411 / 2, T)=5.46 \times 10^{-15} \times n_{\mathrm{H}}\left(\frac{T}{5000}\right)^{0.416} \\
& \text { - } \operatorname{RbI}\left(5 \mathrm{~s}^{2} \mathrm{~S}_{1 / 2}\right)-\mathrm{HI}\left(1 \mathrm{~s}^{2} \mathrm{~S}_{1 / 2}\right) \text { : } \\
& D^{1}(511 / 2, T)=5.82 \times 10^{-15} \times n_{\mathrm{H}}\left(\frac{T}{5000}\right)^{0.414} \\
& \text { - Cs I }\left(6 \mathrm{~s}^{2} \mathrm{~S}_{1 / 2}\right)-\mathrm{HI}\left(1 \mathrm{~s}^{2} \mathrm{~S}_{1 / 2}\right) \text { : } \\
& D^{1}(611 / 2, T)=6.40 \times 10^{-15} \times n_{\mathrm{H}}\left(\frac{T}{5000}\right)^{0.419} .
\end{aligned}
$$

Calculations were performed using the accurate potential energy curves $V^{1} \Sigma$ and $V^{3} \Sigma$ of Geum et al. (2001) for the LiH, $\mathrm{NaH}, \mathrm{KH}, \mathrm{RbH}$ and $\mathrm{CsH}$ ground states. These potentials were obtained by ab initio quantum chemistry methods. We employed our semi-classical description of the collision dynamics. Destruction of orientation rates for the ground states of alkali-atoms for a typical temperature $T=5000 \mathrm{~K}$ are shown in Fig. 4 together with our general results given as function of $n^{*}$. In Fig. 4 we also show the rate of destruction of orientation for the ground state of $\mathrm{NaI}$ at $T=5000 \mathrm{~K}$ obtained from a fully quantal approach (Kerkeni et al. 2000b). In Fig. 5, we show the curves for the Li I, Na I, K I, Rb I and Cs I ground states for our depolarization rates and the rates using the ab initio potentials of Geum et al. (2001), as a function of the local

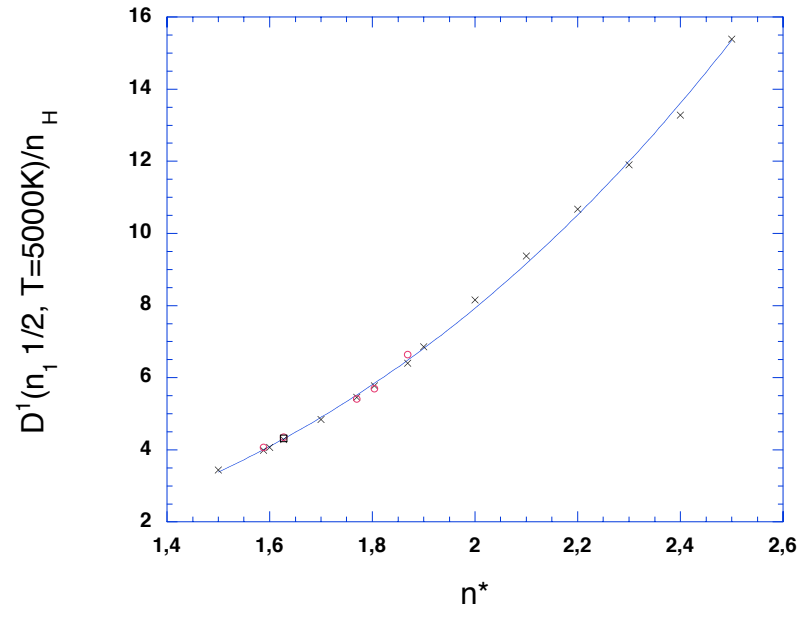

Fig. 4. Plot of depolarization rates of s-states, per unit H-atom density, for local temperature $T=5000 \mathrm{~K}$ with effective principal quantum number $n^{*}$. Full lines: the power law relation of Eq. (19); circles: results for alkali-atoms using Geum et al. (2001) potentials; crosses: our general results; squares: full quantal calculation for Na I (Kerkeni et al. 2000b). Note that it is very difficult to discern differences between the results for the $\mathrm{NaI}\left(n^{*}=1.627\right)$. The rates are given in $10^{-15} \mathrm{rad} \mathrm{m}^{3} \mathrm{~s}^{-1}$.

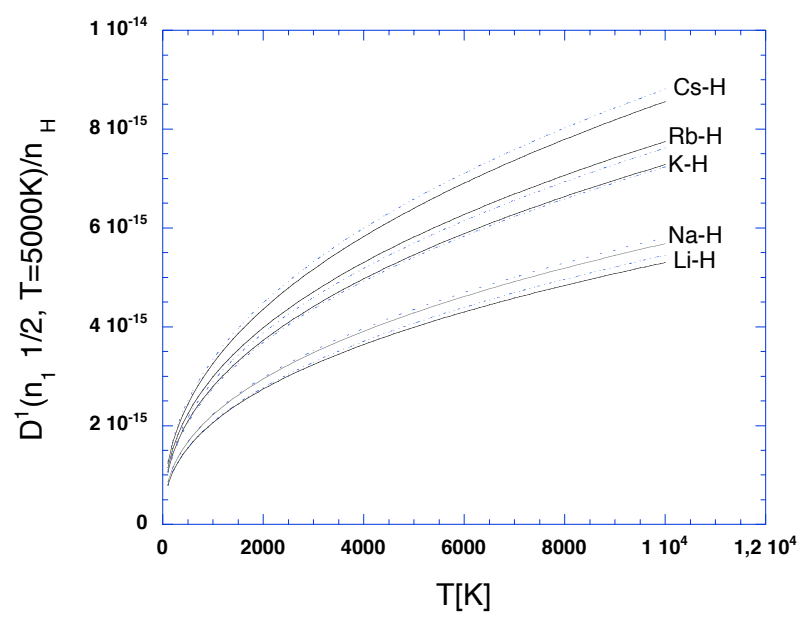

Fig. 5. Destruction of orientation rates for ground levels of $\mathrm{Li}, \mathrm{K}, \mathrm{Rb}$, and Cs atoms per unit $\mathrm{H}$-atom density, $D^{1}\left(n_{1} 1 / 2, T\right) / n_{\mathrm{H}}$, as a function of temperature $T$. Full lines: our results; dotted lines: our calculations using potentials from Geum et al. (2001). The rates are given in $10^{-15} \mathrm{rad} \mathrm{m}^{3} \mathrm{~s}^{-1}$.

temperature $T$. Besides, the curves for the Na I ground state are shown in Fig. 6, along with the full quantum depolarization rates (Kerkeni et al. 2000b). We note that, our general results, using MSMAU potentials and the semi-classical description of the dynamics, are in very good agreement with the rates obtained from the ab initio potentials of Geum et al. (2001) and the full quantum chemistry results of Kerkeni et al. (2000b). In particular for $\mathrm{Na}$ I atom ground level, at $T=5000 \mathrm{~K}$, the percentage error with respect to quantum chemistry depolarization rate of Kerkeni et al. (2000b) is less than $1 \%$.

We conclude from Fig. 5 that the MSMAU potentials, obtained from general treatement employing perturbation theory and the Unsöld approximation, are in sufficient agreement with 


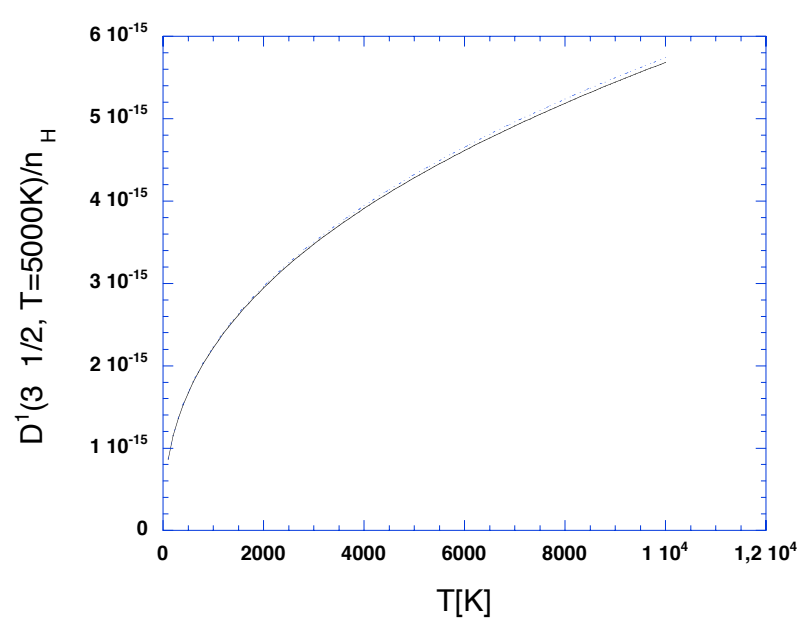

Fig. 6. Depolarization rates per unit H-atom density, $D^{1}(31 / 2, T) / n_{\mathrm{H}}$ for the Na I ground state as a function of temperature. Full lines: our general results; dotted lines: fully quantal calculations (Kerkeni et al. $2000 \mathrm{~b}$ ). The rates are given in $10^{-15} \mathrm{rad} \mathrm{m}^{3} \mathrm{~s}^{-1}$.

the sophisticated Geum et al. (2001) potentials to provide accurate depolarization rates. In addition, the destruction of orientation rate for ground state of $\mathrm{Na}$ I obtained in this work is very close to the one given in Kerkeni et al. (2000b) obtained from fully quantum calculations. Thus, the close-coupling approach with full quantum dynamics is not necessary for reasonable precise depolarization rates calculations, and the collision may be adequately modelled with the classical straight path approximation. We notice that the differences between our rates using the MSMAU potentials and those from quantum chemistry potentials increase when the temperatures of the medium increase. In fact, when the temperatures increase, the interatomic separations playing the major role in the depolarization phenomenon decreases. When the interatomic separations decrease the differences between the MSMAU potentials and quantum chemistry potentials increase.

\section{Depolarization and polarization transfer rates of complex atoms}

\subsection{Theory}

Papers I, II, III, IV have been concerned with simple atoms/ions for which the electronic configurations have only one valence electron in non-spherically symmetric state $(l \neq 0)$ above a filled-subshell (for example: singly ionised calcium in the state $1 s^{2} 2 s^{2} 2 p^{6} 4 p^{2} P_{3 / 2}$ ) or contain, in addition to the valence electron, an electron in a s-state (for example: neutral calcium in the state $\left.1 s^{2} 2 s^{2} 2 p^{6} 4 s 4 p^{1} P_{1}\right)$.

We now consider the problem of the calculation of depolarization polarization transfer rates for complex atoms (Fe I, Ti I, ...). The electronic configuration of the complex atom/ion has one valence electron above an incomplete (open) subshell which has a non-zero angular momentum. We assume that only the valence electron can undergo collisions with hydrogen atoms (the frozen core approximation). For more details of our approach concerning with complex atoms we refer to Paper V and references therein. As regards the solar lines commonly observed in the photosphere originating from complex atoms, the relevant states are usually well described within the approximation of $L-S$ coupling (see, e.g., Sobelman 1992; Landi Degl'Innocenti 1982). Having the scattering matrix between the Zeeman sublevels of complex atoms $\left\langle J M_{J}\left|S_{\text {sc }}\right| J^{\prime} M_{J}^{\prime}\right\rangle$ (see Eq. (7) of Paper V), we have to perform an average of the transitions probabilities over all possible orientations since collisions are isotropic. All rates are obtained after integration over impact parameters and velocities as described in our previous papers. This is a direct way following that the calculations of rates involving complex atoms must proceed line by line using our numerical code.

We propose an indirect and more practical way that consists in the interpolation of the values of rates given for simple atoms. As in Paper V, we denote by $\boldsymbol{L}_{\mathbf{c}}$ the total orbital angular momentum of the core of the atom and by $\boldsymbol{S}_{\mathrm{c}}$ its total spin. Because the orbital angular momentum of the valence electron in a spherically symmetric state is $\boldsymbol{l}=\mathbf{0}$, the total orbital momentum of the atom is $\boldsymbol{L}=\boldsymbol{L}_{\mathbf{c}}+\boldsymbol{l}=\boldsymbol{L}_{\mathbf{c}}$. The total spin is $\boldsymbol{S}=\boldsymbol{S}_{\mathrm{c}}+\boldsymbol{s}$ and $\boldsymbol{J}=\boldsymbol{L}+\boldsymbol{S}=\boldsymbol{L}_{\mathrm{c}}+\boldsymbol{S}$ is the total angular momentum. We now consider the expansion of the matrix of the depolarization and polarization transfer associated with the sublevels $\left|n_{1} L J\right\rangle$ over the irreducible tensorial operator basis ${ }^{L_{\mathrm{c}} S J} T_{q}^{k}$. The depolarization rate matrix for the state $\left|n_{1} S\right\rangle$ can be expanded in terms of the tensors ${ }^{S} T_{q_{1}}^{k_{1}}$ and similarly for the state $\left|n_{1} L_{\mathrm{c}}\right\rangle$ one can expand the depolarization rate matrix in the tensor basis ${ }^{L_{\mathrm{c}}} T_{q_{2}}^{k_{2}}$. That is, the total spin and orbital angular momentum of the core of the complex atom are considered independently. The physical contents of the combined basis ${ }^{L_{\mathrm{c}} S J} T_{q}^{k}$ are completely determined by the product ${ }^{L_{\mathrm{c}}} T_{q_{2}}^{k_{2}} \otimes{ }^{S} T_{q_{1}}^{k_{1}}$ and one can easily show that:

$$
\begin{aligned}
& { }^{L_{\mathrm{c}} S J} T_{q}^{k}=(2 J+1) \sum_{k_{1}, q_{1}, k_{2}, q_{2}} \sqrt{\left(2 k_{1}+1\right)\left(2 k_{2}+1\right)} \\
& \times \sqrt{(2 k+1)}(-1)^{k_{1}-k_{2}+q}\left(\begin{array}{ccc}
k_{1} & k_{2} & k \\
q_{1} & q_{2} & -q
\end{array}\right) \\
& \times\left\{\begin{array}{lll}
L_{\mathrm{c}} & S & J \\
L_{\mathrm{c}} & S & J \\
k_{2} & k_{1} & k
\end{array}\right\}{ }^{L_{\mathrm{c}} L_{\mathrm{c}}} T_{q_{1}}^{k_{1}} S S^{\prime} T_{q_{2}}^{k_{2}},
\end{aligned}
$$

where the term in brackets \{\} (3 lines $\times 3$ columns) denotes a Wigner 9 $j$-coefficient (Messiah 1961). By expansion of the depolarization matrix $\left(J=J^{\prime}\right)$ and polarization transfer matrix $\left(J \neq J^{\prime}\right)$ over the tensorial basis and by taking into account the summation rule,

$(2 k+1) \sum_{q_{1}, q_{2}}\left(\begin{array}{ccc}k_{1} & k_{2} & k \\ q_{1} & q_{2} & -q\end{array}\right)^{2}=1$,

one can show that:

$$
\begin{aligned}
& \zeta^{k}\left(n_{1} L J \rightarrow n_{1} L J^{\prime}, T\right)\left(J=J^{\prime} \text { and } J \neq J^{\prime}\right)= \\
& (2 J+1)\left(2 J^{\prime}+1\right) \sum_{k_{1}}\left(2 k_{1}+1\right) \zeta^{k_{1}}\left(n_{1} S, T\right) \\
& \times \sum_{k_{2}}\left(2 k_{2}+1\right)\left\{\begin{array}{l}
L_{\mathrm{c}} S J \\
L_{\mathrm{c}} S J \\
k_{2} k_{1} k
\end{array}\right\}\left\{\begin{array}{l}
L_{\mathrm{c}} S J^{\prime} \\
L_{\mathrm{c}} S J^{\prime} \\
k_{2} k_{1} k
\end{array}\right\}
\end{aligned}
$$


Formally, Eq. (24) is the same as Eq. (4.6) of Nienhuis (1976) or Eq. (4.48) of Omont (1977). $\zeta^{k_{1}}\left(n_{1} S, T\right)$ are the spherical tensor components of the elastic collisional rates acting in the part of the complex atom associated to its spin. Thus, $D^{k_{1}}\left(n_{1} S, T\right)=\zeta^{0}\left(n_{1} S, T\right)-\zeta^{k_{1}}\left(n_{1} S, T\right)$ is the depolarizing rate of the level $\left|n_{1} S\right\rangle$. Note that there are no collisional depolarizing rates associated to the core of the complex atom which is ignored during the collision; the order $k_{2}$ inside the core is not destroyed by the isotropic collisions. The phenomenon of the collisional depolarization of the combined system (i.e. the complex atom) is reduced to the depolarization of the spin, the depolarization rate of the level $\left|n_{1} L J\right\rangle$ of the complex atom is given by:

$D^{k}\left(n_{1} L J, T\right)=\zeta^{0}\left(n_{1} L J, T\right)-\zeta^{k}\left(n_{1} L J, T\right)$.

If $k=0$, as usual, the destruction rate of population is zero which states that the elastic collisions (i.e. $J=J^{\prime}$ ) do not alter the total population of the level $\left(n_{1} L J\right)$. In the same way we write the expression for the depolarization rate $\zeta^{k_{1}}\left(n_{1} S, T\right)$ as a linear combination of the depolarization rates $\zeta^{k_{3}}\left(n_{1} s, T\right)$, where $\left|n_{1} s\right\rangle$ is the state of the valence electron of the perturbed atom/ion,

$$
\begin{aligned}
\zeta^{k_{1}}\left(n_{1} S, T\right)= & (2 S+1)^{2} \sum_{k_{3}} \zeta^{k_{3}}\left(n_{1} s, T\right) \sum_{k_{4}}\left(2 k_{4}+1\right) \\
& \times\left\{\begin{array}{ccc}
S & S_{\mathrm{c}} & S \\
S & S_{\mathrm{c}} & S \\
k_{3} & k_{4} & k_{1}
\end{array}\right\} .
\end{aligned}
$$

From Eqs. (24) and (26), we find that:

$$
\begin{aligned}
\zeta^{k}\left(n L J \rightarrow n L J^{\prime}, T\right)= & \sum_{k_{3}=0}^{1} \zeta^{k_{3}}\left(n_{1} s, T\right) \mathcal{A}^{k}\left(L J \rightarrow L J^{\prime} ; k_{3}\right) \\
= & \zeta^{0}\left(n_{1} s, T\right) \mathcal{A}^{k}\left(L J \rightarrow L J^{\prime} ; 0\right) \\
& +\zeta^{1}\left(n_{1} s, T\right) \mathcal{A}^{k}\left(L J \rightarrow L J^{\prime} ; 1\right)
\end{aligned}
$$

where,

$$
\begin{aligned}
\mathcal{A}^{k}\left(L J \rightarrow L J^{\prime} ; k_{3}\right)= & \left(2 k_{3}+1\right)(2 J+1)\left(2 J^{\prime}+1\right)(2 S+1)^{2} \\
& \times\left(2 k_{4}+1\right) \sum_{k_{1}, k_{2}, k_{4}}\left(2 k_{1}+1\right)\left(2 k_{2}+1\right) \\
& \times\left\{\begin{array}{ccc}
S & S_{\mathrm{c}} & S \\
S & S_{\mathrm{c}} & S \\
k_{3} & k_{4} & k_{1}
\end{array}\right\}\left\{\begin{array}{lll}
L_{\mathrm{c}} & S & J \\
L_{\mathrm{c}} & S & J \\
k_{2} & k_{1} & k
\end{array}\right\} \\
& \times\left\{\begin{array}{lll}
L_{\mathrm{c}} & S & J^{\prime} \\
L_{\mathrm{c}} & S & J^{\prime} \\
k_{2} & k_{1} & k
\end{array}\right\}
\end{aligned}
$$

$\mathcal{A}^{k}\left(L J \rightarrow L J ; k_{3}\right)$ is the depolarization coefficient of the state $\left|n_{1} L J\right\rangle$ of the complex atom. The depolarizing rate of the level $\left|n_{1} L J\right\rangle$ at a temperature $T$ of the medium is:

$$
D^{k}\left(n_{1} L J, T\right)=\zeta^{0}\left(n_{1} L J, T\right)-\zeta^{k}\left(n_{1} L J, T\right) .
$$

Because, in our approach, the collision do not affect the state of internal electrons and so that $\boldsymbol{L}_{\mathrm{c}}$ and $\boldsymbol{S}_{\mathrm{c}}$ are conserved, it is easy to show that:

$\zeta^{0}\left(n_{1} L J, T\right)=\zeta^{0}\left(n_{1} s, T\right)$.
Thus, taking into account Eq. (27),

$$
\begin{aligned}
D^{k}\left(n_{1} L J, T\right)= & \zeta^{0}\left(n_{1} s, T\right) \times\left[1-\mathcal{A}^{k}(L J \rightarrow L J ; 0)\right] \\
& -\zeta^{1}\left(n_{1} s, T\right) \times A^{k}(L J \rightarrow L J ; 1)
\end{aligned}
$$

where $\zeta^{0}\left(n_{1} s, T\right)$ and $\zeta^{1}\left(n_{1} s, T\right)$ are given by Eqs. (20) and (21) if one takes $n^{*}=\left[2 E_{\infty}\right]^{-1 / 2}, E_{\infty}$ is the binding energy of the valence electron of the complex atom in atomic units. Note, if it assumed, as we have done, that the internal core electrons are not perturbed by the collisions, the interaction potential appropriate for calculation of the depolarizing collisions of the state $\left|n_{1} L J\right\rangle$ is the same as that for the depolarizing collisions of state $\left|n_{1} s\right\rangle$. The same results have been obtained in Paper V when we expressed the scattering matrix elements of complex atoms colliding with hydrogen atoms as a linear combination of those of simple atoms colliding with hydrogen atoms (Paper V, Eq. (7)). Concerning the problem of how important is the dpolarization rate $D^{k}\left(n_{1} L J, T\right)$ of complex atoms compared to $D^{k_{3}}\left(n_{1} s, T\right)$, there is no common answer for whatever case and one have to calculate the depolarization coefficient $A^{k}\left(L J \rightarrow L J^{\prime} ; k_{3}\right)$ for each level. Obviously, one can easily compute $A^{k}\left(L J \rightarrow L J^{\prime} ; k_{3}\right)$ by programming Eq. (28). We notice that the total depolarizing effect of collisions for a state of a complex atom is expanded into a series of non-zero depolarization rates associated with ranks $0<k<2 J$ while there is only one non-zero depolarizing rate, namely destruction of orientation $k_{3}=1$, for the s-state in the simple atom. This point may be worthy of further study.

We point out two interesting particular cases of Eq. (28):

- Spin unpolarized core, i.e. $k_{2}=0$ and $k_{4}=0$ : Eq. (28) is then reduced to

$$
\begin{aligned}
& \mathcal{A}^{k}\left(L J \rightarrow L J^{\prime} ; k_{3}\right)=\delta_{k_{3}, k}(2 J+1)\left(2 J^{\prime}+1\right) \\
& \times \frac{(2 S+1)^{2}}{\left(2 S_{\mathrm{c}}+1\right)\left(2 L_{\mathrm{c}}+1\right)}\left\{\begin{array}{l}
S S_{\mathrm{c}} \\
s S k_{3}
\end{array}\right\}^{2}\left\{\begin{array}{l}
S J L_{\mathrm{c}} \\
S J k_{3}
\end{array}\right\}\left\{\begin{array}{l}
S J^{\prime} L_{\mathrm{c}} \\
S J^{\prime} k_{3}
\end{array}\right\}
\end{aligned}
$$

\{ \} (2 lines $\times 2$ columns) is a $6 j$-coefficient (Messiah 1961). $-L_{\mathrm{c}}=0$ and $S_{\mathrm{c}}=0$ (simple atoms): we retrieve from the general expression of equation (28) the depolarization rate of the particular case of simple atoms in a spherically symmetric state because,

$$
\mathcal{A}^{k}\left(L J \rightarrow L J^{\prime} ; k_{3}\right)=\delta(s, J) \delta\left(s, J^{\prime}\right) \delta\left(k_{3}, k\right)
$$

so that,

$$
\zeta^{k}\left(n L J \rightarrow n L J^{\prime}, T\right)=\zeta^{k_{3}}\left(n_{1} s, T\right) \delta(s, J) \delta\left(s, J^{\prime}\right) \delta\left(k_{3}, k\right)
$$

and

$$
D^{k}(n L J, T)=D^{k_{3}}\left(n_{1} s, T\right) \delta(s, J) \delta\left(s, J^{\prime}\right) \delta\left(k_{3}, k\right) .
$$

\subsection{Example of calculation}

In order to illustrate how our theory allows us to obtain depolarization and polarization transfer rates of levels of complex atoms, we consider the particular cases of the lower states involved in the transitions of $\mathrm{Ti}$ I of multiplets $42\left(3 \mathrm{~d}^{3}\left({ }^{4} \mathrm{~F}\right) 4 \mathrm{~s}\left({ }^{5} \mathrm{~F}\right)\right)$ and $145\left(3 \mathrm{~d}^{3}\left({ }^{4} \mathrm{P}\right) 4 \mathrm{~s}\left({ }^{5} \mathrm{P}\right)\right)$ 
Table 2. $\mathcal{A}^{k}\left(n_{1} L J \rightarrow n_{1} L J^{\prime}\right)\left(J=J^{\prime}\right)$ for the lower state of multiplet 42 of neutral titanium Ti I.

\begin{tabular}{|c|c|c|c|c|}
\hline$J$ & $J^{\prime}$ & $k$ & $\mathcal{A}^{k}\left(n_{1} L J \rightarrow n_{1} L J^{\prime} ; 0\right)$ & $\mathcal{A}^{k}\left(n_{1} L J \rightarrow n_{1} L J^{\prime} ; 1\right)$ \\
\hline 1 & 1 & 0 & $6.43 \times 10^{-2}$ & $1.46 \times 10^{-1}$ \\
\hline 1 & 1 & 1 & $7.29 \times 10^{-2}$ & $2.42 \times 10^{-1}$ \\
\hline 1 & 1 & 2 & $6.94 \times 10^{-2}$ & $4.87 \times 10^{-1}$ \\
\hline 2 & 2 & 0 & $2.68 \times 10^{-2}$ & $5.06 \times 10^{-1}$ \\
\hline 2 & 2 & 1 & $3.75 \times 10^{-2}$ & $4.50 \times 10^{-1}$ \\
\hline 2 & 2 & 2 & $7.12 \times 10^{-2}$ & $3.47 \times 10^{-1}$ \\
\hline 2 & 2 & 3 & $1.15 \times 10^{-1}$ & $2.63 \times 10^{-1}$ \\
\hline 2 & 2 & 4 & $4.97 \times 10^{-2}$ & $4.17 \times 10^{-1}$ \\
\hline 3 & 3 & 0 & $6.67 \times 10^{-2}$ & $3.20 \times 10^{-1}$ \\
\hline 3 & 3 & 1 & $6.37 \times 10^{-2}$ & $3.22 \times 10^{-1}$ \\
\hline 3 & 3 & 2 & $5.92 \times 10^{-2}$ & $3.27 \times 10^{-1}$ \\
\hline 3 & 3 & 3 & $5.95 \times 10^{-2}$ & $3.3 \times 10^{-1}$ \\
\hline 3 & 3 & 4 & $7.50 \times 10^{-2}$ & $3.27 \times 10^{-1}$ \\
\hline 3 & 3 & 5 & $1.01 \times 10^{-1}$ & $3.37 \times 10^{-1}$ \\
\hline 3 & 3 & 6 & $5.21 \times 10^{-2}$ & $4.71 \times 10^{-1}$ \\
\hline 4 & 4 & 0 & $8.57 \times 10^{-2}$ & $5.78 \times 10^{-2}$ \\
\hline 4 & 4 & 1 & $8.37 \times 10^{-2}$ & $7.59 \times 10^{-2}$ \\
\hline 4 & 4 & 2 & $7.83 \times 10^{-2}$ & $1.13 \times 10^{-1}$ \\
\hline 4 & 4 & 3 & $6.89 \times 10^{-2}$ & $1.67 \times 10^{-1}$ \\
\hline 4 & 4 & 4 & $5.87 \times 10^{-2}$ & $2.36 \times 10^{-1}$ \\
\hline 4 & 4 & 5 & $5.62 \times 10^{-2}$ & $3.10 \times 10^{-1}$ \\
\hline 4 & 4 & 6 & $7.02 \times 10^{-2}$ & $3.80 \times 10^{-1}$ \\
\hline 4 & 4 & 7 & $9.20 \times 10^{-2}$ & $4.58 \times 10^{-1}$ \\
\hline 4 & 4 & 8 & $6.00 \times 10^{-2}$ & $6.11 \times 10^{-1}$ \\
\hline 5 & 5 & 0 & $6.55 \times 10^{-3}$ & $8.04 \times 10^{-3}$ \\
\hline 5 & 5 & 1 & $9.64 \times 10^{-3}$ & $1.02 \times 10^{-2}$ \\
\hline 5 & 5 & 2 & $1.64 \times 10^{-2}$ & $1.51 \times 10^{-2}$ \\
\hline 5 & 5 & 3 & $2.78 \times 10^{-2}$ & $2.42 \times 10^{-2}$ \\
\hline 5 & 5 & 4 & $4.46 \times 10^{-2}$ & $4.04 \times 10^{-2}$ \\
\hline 5 & 5 & 5 & $6.71 \times 10^{-2}$ & $6.90 \times 10^{-2}$ \\
\hline 5 & 5 & 6 & $9.31 \times 10^{-2}$ & $1.19 \times 10^{-1}$ \\
\hline 5 & 5 & 7 & $1.17 \times 10^{-1}$ & $2.06 \times 10^{-1}$ \\
\hline 5 & 5 & 8 & $1.27 \times 10^{-1}$ & $3.54 \times 10^{-1}$ \\
\hline 5 & 5 & 9 & $10^{-1}$ & $3 / 5$ \\
\hline 5 & 5 & 10 & 0 & 1 \\
\hline
\end{tabular}

(Manso Sainz \& Landi Degl'Innocenti 2002). These multiplets show high degrees of polarization in the second solar spectrum. For the first case, $\left(3 \mathrm{~d}^{3}\left({ }^{4} \mathrm{~F}\right) 4 \mathrm{~s}\left({ }^{5} \mathrm{~F}\right)\right)$, we have $L_{\mathrm{c}}=3, s=1 / 2$, $L=3, S=2, S_{\mathrm{c}}=3 / 2$ and $J$ takes on the values $1,2,3$, 4 and 5 . In the second case of the $\left(3 \mathrm{~d}^{3}\left({ }^{4} \mathrm{P}\right) 4 \mathrm{~s}\left({ }^{5} \mathrm{P}\right)\right)$ multiplet $L_{\mathrm{c}}=1, s=1 / 2, L=1, S=2, S_{\mathrm{c}}=3 / 2$ and $J$ takes on the values 1, 2 and 3. By application of Eqs. (20), (21) and (27), the depolarizing rates are given by:

$$
\begin{aligned}
D^{k}\left(n_{1} L J, T\right)= & \left(\left[31.214-53.630 \times n^{*}+25.177 \times n^{*^{2}}\right]\right. \\
& \times\left[1-\mathcal{A}^{k}(L J \rightarrow L J ; 0)\right] \\
& +\left[27.234-48.575 \times n^{*}+21.88 \times n^{*^{2}}\right] \\
& \left.\times\left[\mathcal{A}^{k}(L J \rightarrow L J ; 1)\right]\right) \\
& \times 10^{-15} n_{\mathrm{H}}\left(\frac{T}{5000}\right)^{0.416}\left(\operatorname{rad~s}^{-1}\right),
\end{aligned}
$$

and the polarization transfer rates:

$$
\begin{aligned}
& \zeta^{k}\left(n_{1} L J \rightarrow n_{1} L J^{\prime}, T\right)\left(J \neq J^{\prime}\right)= \\
& \left(\left[31.214-53.630 \times n^{*}+25.177 \times n^{*^{2}}\right] \times\left[\mathcal{A}^{k}\left(L J \rightarrow L J^{\prime} ; 0\right)\right]\right. \\
& \left.+\left[27.234-48.575 \times n^{*}+21.88 \times n^{*^{2}}\right] \times\left[\mathcal{A}^{k}\left(L J \rightarrow L J^{\prime} ; 1\right)\right]\right) \\
& \times 10^{-15} n_{\mathrm{H}}\left(\frac{T}{5000}\right)^{0.416}\left(\mathrm{rad} \mathrm{s}^{-1}\right)
\end{aligned}
$$

where for the lower levels of the multiplets 42 and $145, n^{*}=$ 1.504 and 1.636 respectively. Tables $2-4$ show all non-zero depolarization coefficients $\mathcal{A}^{k}\left(L J \rightarrow L J^{\prime} ; k_{3}\right)$ for multiplets 42 and 145 allowing the calculation of all non-zero depolarization and polarization transfer rates. In particular, for the term levels $\left({ }^{5} \mathrm{~F}_{1}\right)(J=1)$ and $\left({ }^{5} \mathrm{P}_{1}\right)(J=1)$ of the multiplets 42 and 145 respectively, one can readily get the analytical 
Table 3. $\mathcal{A}^{k}\left(n_{1} L J \rightarrow n_{1} L J^{\prime}\right)\left(J \neq J^{\prime}\right)$ for the lower state of multiplet 42 of neutral titanium Ti I.

\begin{tabular}{ccccc}
\hline \hline$k$ & $J$ & $J^{\prime}$ & $\mathcal{A}^{k}\left(n_{1} L J \rightarrow n_{1} L J^{\prime} ; 0\right)$ & $\mathcal{A}^{k}\left(n_{1} L J \rightarrow n_{1} L J^{\prime} ; 1\right)$ \\
\hline 1 & 2 & 0 & $-4.15 \times 10^{-2}$ & $-2.57 \times 10^{-1}$ \\
1 & 2 & 1 & $-1.44 \times 10^{-2}$ & $-2.83 \times 10^{-1}$ \\
1 & 2 & 2 & $2.66 \times 10^{-2}$ & $-2.83 \times 10^{-1}$ \\
2 & 3 & 0 & $4.23 \times 10^{-2}$ & $-4.01 \times 10^{-1}$ \\
2 & 3 & 1 & $1.34 \times 10^{-2}$ & $-3.48 \times 10^{-1}$ \\
2 & 3 & 2 & $2.00 \times 10^{-2}$ & $-2.71 \times 10^{-1}$ \\
2 & 3 & 3 & $-2.12 \times 10^{-2}$ & $-2.13 \times 10^{-1}$ \\
2 & 3 & 4 & $2.65 \times 10^{-2}$ & $-1.88 \times 10^{-1}$ \\
3 & 4 & 0 & $-7.56 \times 10^{-2}$ & $-1.10 \times 10^{-1}$ \\
3 & 4 & 1 & $-5.67 \times 10^{-2}$ & $-1.35 \times 10^{-1}$ \\
3 & 4 & 2 & $-2.84 \times 10^{-2}$ & $-1.74 \times 10^{-1}$ \\
3 & 4 & 3 & $-3.06 \times 10^{-3}$ & $-2.08 \times 10^{-1}$ \\
3 & 4 & 4 & $1.29 \times 10^{-2}$ & $-2.24 \times 10^{-1}$ \\
3 & 4 & 5 & $2.46 \times 10^{-2}$ & $-2.18 \times 10^{-1}$ \\
3 & 4 & 6 & $3.96 \times 10^{-2}$ & $-1.84 \times 10^{-1}$ \\
4 & 5 & 0 & $-2.37 \times 10^{-2}$ & $-1.63 \times 10^{-2}$ \\
4 & 5 & 1 & $-2.76 \times 10^{-2}$ & $-1.94 \times 10^{-2}$ \\
4 & 5 & 2 & $-3.33 \times 10^{-2}$ & $-2.73 \times 10^{-2}$ \\
4 & 5 & 3 & $-3.72 \times 10^{-2}$ & $-4.26 \times 10^{-2}$ \\
4 & 5 & 4 & $-3.37 \times 10^{-2}$ & $-6.88 \times 10^{-2}$ \\
4 & 5 & 5 & $-1.75 \times 10^{-2}$ & $-1.08 \times 10^{-1}$ \\
4 & 5 & 6 & $1.44 \times 10^{-2}$ & $-1.56 \times 10^{-1}$ \\
4 & 5 & 7 & $5.71 \times 10^{-2}$ & $-2.02 \times 10^{-1}$ \\
4 & 5 & 8 & $8.72 \times 10^{-2}$ & $-2.04 \times 10^{-1}$ \\
\hline & & & &
\end{tabular}

expressions of the destruction rates of alignment $(k=2)$ in $\operatorname{rad~s}^{-1}$ for $100 \leq T \leq 10000 \mathrm{~K}$ :

$\operatorname{Ti} \mathrm{I}\left(3 \mathrm{~d}^{3}\left({ }^{4} \mathrm{~F}\right) 4 \mathrm{~s}\left({ }^{5} \mathrm{~F}_{1}\right)\right)-\mathrm{HI}(1 \mathrm{~s})$ :

$D^{2}(431, T)=8.77 \times 10^{-15} n_{\mathrm{H}}\left(\frac{T}{5000}\right)^{0.416}$,

Ti I $\left(3 d^{3}\left({ }^{4} \mathrm{P}\right) 4 \mathrm{~s}\left({ }^{5} \mathrm{P}_{1}\right)\right)-\mathrm{H} \mathrm{I}(1 \mathrm{~s})$ :

$D^{2}(411, T)=14.30 \times 10^{-15} n_{\mathrm{H}}\left(\frac{T}{5000}\right)^{0.416}$.

\section{Hyperfine depolarization and polarization transfer rates}

The existence of complicated structures of the observed polarization profiles of some lines of the second solar spectrum, such as Li I $6707 \AA$, Ba II $4554 \AA$, Na I $5896 \AA$, and K I doublet (at $7699 \AA$ and $7665 \AA$ ), etc, should be explained in terms of hyperfine structure splitting. To quantitatively explain these structures, collisional depolarizing and polarization transfer rates for the hyperfine sublevels should be taken into account in the polarized radiative transfer models. If the atom/ion possesses nuclear angular momentum $I$, the total angular momentum of the atom is denoted with $F$ where $\boldsymbol{F}=\boldsymbol{J}+\boldsymbol{I}$. Coupling of $J$ with $I$ results in the hyperfine splitting of each $J$ into levels, $F=|J-I|,|J-I+1|, \ldots, J+I$. As has been mentioned in Sect. 2, to a very good approximation, $\boldsymbol{I}$ is conserved during the collision processes allowing the $S_{\mathrm{sc}}$-matrix is diagonal in $I$ and its elements do not depend on $M_{I}$. Then according to the same procedure as the one used in Sect. 7, it is simple to deduce hyperfine depolarizing and polarization transfer rates for simple/complex atoms/ions from our depolarizing rates of fine structure levels of simple/complex atoms/ions given in Sect. 6. The linear combinations have been given by Eqs. (4.6) of Nienhuis (1976) or (4.48) of Omont (1977). In fact, the hyperfine polarization transfer rates $\zeta^{k}\left(n_{1} J I F \rightarrow n_{1} J I F^{\prime}, T\right)$ are readily obtained by carrying out the formal substitutions: $J \rightarrow$ $F, L_{\mathrm{c}} \rightarrow J$ and $S \rightarrow I$ on the Eq. (24). The hyperfine depolarizing rates are $D^{k}\left(n_{1} J I F, T\right)=\zeta^{0}\left(n_{1} J I F, T\right)-\zeta^{k}\left(n_{1} J I F, T\right)$.

\section{Conclusion}

In this paper we have proposed a general treatment including the effects of spin to calculate depolarization rates of s-states due to isotropic collisions with neutral hydrogen. Rates for the ground states of alkali metals (Li I, Na I, K I, Rb I and Cs I) have been calculated as an application of the theory and for comparison. The resulting destruction rate of orientation of the $\mathrm{Na}$ I ground state is in excellent agreement with that found by full quantum chemistry approach of Kerkeni et al. (2000b). In fact, at $T=5000 \mathrm{~K}$ the difference is less than $1 \%$. We studied the variation of the depolarizing rates with effective principal quantum number $n^{*}$ ranging from 1.3 to 3 . and we found that the behaviour of the depolarization rates with $n^{*}$ obeys a simple power law. We showed that the depolarization and polarization transfer rates of levels of complex atoms may be derived from calculations of rates associated to simple atoms. As application, we tabulate results for all non-zero depolarization and polarization transfer rates of the lower levels of multiplets 42 
Table 4. Depolarization coefficients $\mathcal{A}^{k}\left(n_{1} L J \rightarrow n_{1} L J^{\prime}\right)\left(J=J^{\prime}\right.$ and $\left.J \neq J^{\prime}\right)$ for the lower state of multiplet 145 of neutral titanium Ti I.

\begin{tabular}{ccccc}
\hline \hline$k$ & $J$ & $J^{\prime}$ & $\mathcal{A}^{k}\left(n_{1} L J \rightarrow n_{1} L J^{\prime} ; 0\right)$ & $\mathcal{A}^{k}\left(n_{1} L J \rightarrow n_{1} L J^{\prime} ; 1\right)$ \\
\hline 1 & 1 & 0 & 0 & $1.31 \times 10^{-2}$ \\
1 & 1 & 1 & $1.05 \times 10^{-1}$ & $1.31 \times 10^{-2}$ \\
1 & 1 & 2 & $6.30 \times 10^{-2}$ & $6.51 \times 10^{-1}$ \\
2 & 2 & 0 & 0 & $2.19 \times 10^{-2}$ \\
2 & 2 & 1 & $5.83 \times 10^{-2}$ & $7.29 \times 10^{-3}$ \\
2 & 2 & 2 & $7.50 \times 10^{-2}$ & $10^{-1}$ \\
2 & 2 & 3 & $10^{-1}$ & $2.94 \times 10^{-1}$ \\
2 & 2 & 4 & $5.5 \times 10^{-2}$ & $7.32 \times 10^{-1}$ \\
3 & 3 & 0 & 0 & $2.50 \times 10^{-3}$ \\
3 & 3 & 1 & $3.33 \times 10^{-3}$ & $6.67 \times 10^{-3}$ \\
3 & 3 & 2 & $1.80 \times 10^{-2}$ & $1.60 \times 10^{-2}$ \\
3 & 3 & 3 & $5.67 \times 10^{-2}$ & $3.83 \times 10^{-2}$ \\
3 & 3 & 4 & $1.22 \times 10^{-1}$ & $1.08 \times 10^{-1}$ \\
3 & 3 & 5 & $1.67 \times 10^{-1}$ & $1 / 3$ \\
3 & 3 & 6 & 0 & 1 \\
1 & 2 & 0 & 0 & $-1.69 \times 10^{-2}$ \\
1 & 2 & 1 & $-7.83 \times 10^{-2}$ & $9.78 \times 10^{-3}$ \\
1 & 2 & 2 & $6.87 \times 10^{-2}$ & $-2.35 \times 10^{-1}$ \\
2 & 3 & 0 & 0 & $-7.39 \times 10^{-3}$ \\
2 & 3 & 1 & $-1.39 \times 10^{-2}$ & $-6.97 \times 10^{-3}$ \\
2 & 3 & 2 & $-3.67 \times 10^{-2}$ & $-1.22 \times 10^{-2}$ \\
2 & 3 & 3 & $-2.58 \times 10^{-2}$ & $-6.13 \times 10^{-2}$ \\
2 & 3 & 4 & $8.24 \times 10^{-2}$ & $-1.94 \times 10^{-1}$ \\
\hline & & & &
\end{tabular}

and 145 of neutral titanium. In addition, we outlined how the data may also be used to calculate depolarization rates for hyperfine structure states.

Acknowledgements. M.D. would like to express his gratitude to the Stellar Atmospheres Group in Uppsala University for their kind invitation and hospitality. P.S.B. is supported by the Swedish Research Council (VR). The authors would like to thank S. D. Anstee for provinding numerical code used for the interaction potential computations.

\section{References}

Anstee, S. D. 1992, Ph.D. Thesis, Univ. Queensland

Anstee, S. D., \& O'Mara, B. J. 1991, MNRAS, 253, 549

Anstee, S. D., \& O’Mara, B. J. 1995, MNRAS, 276, 859

Anstee, S. D., O’Mara, B. J., \& Ross, J. E. 1997, MNRAS, 284, 202

Barklem, P. S. 1998, Ph.D. Thesis, Univ. Queensland

Barklem, P. S., \& O'Mara, B. J. 1997, MNRAS, 290, 102

Barklem, P. S., \& O’Mara, B. J., \& Ross, J. E. 1998, MNRAS, 296, 1057

Bommier, V., \& Sahal-Bréchot, S. 1982, Sol. Phys., 78, 157

Carter, W. W. 1949, Phy. Rev., 76, 962

Chipman, D. M., Bowman, J. D., \& Hirschfelder, J. O. 1973, J. Chem. Phys. 59, 2830

Dalgarno, A., \& Williams, D. A. 1962, Proc. Phy. Soc. (London), A69, 57

Derouich, M., Sahal-Bréchot, S., Barklem, P. S., \& O'Mara, B. J. 2003a, A\&A, 404, 763 (Paper I)

Derouich, M., Sahal-Bréchot, S., \& Barklem, P. S. 2003b, A\&A, 409, 369 (Paper II)

Derouich, M., Sahal-Bréchot, S., \& Barklem, P. S. 2004a, A\&A, 414, 369 (Paper III)
Derouich, M., Sahal-Bréchot, S., \& Barklem, P. S. 2004b, A\&A, 426, 707 (Paper IV)

Derouich, M., Sahal-Bréchot, S., \& Barklem, P. S. 2005, A\&A, 434, 779 (Paper V)

EPAPS Document No. E-JCPSA6-115-311132 for tables of the ab initio singlet and triplet potential curves of $\mathrm{LiH}, \mathrm{NaH}, \mathrm{KH}, \mathrm{RbH}$, and $\mathrm{CsH}$. This document may be retrieved via the EPAPS homepage (http://www.aip.org/pubservs/epaps.html) or from ftp.aip.org in the directory /epaps/. See the EPAPS homepage for more information. [EPAPS] first citation in article

Geum, N., Jeung, G.-H., Derevianko, A., Côté, R., \& Dalgarno, A. 2001, J. Ch. Phys., 115, 5984

Gordeyev, E. P., Nikitin, E. E., \& Ovchinnikova, M. Ya. 1969, Can. J. Phys., 47, 1819

Hirschfelder, J. O. 1967a, Chem. Phys. Lett., 1, 325

Hirschfelder, J. O. 1967b, Chem. Phys. Lett., 1, 363

Kerkeni, B. 20002, A\&A, 390, 783

Kerkeni, B., Spielfiedel, A., \& Feautrier, N. 2000a, A\&A, 358, 373

Kerkeni, B., Spielfiedel, A., \& Feautrier, N. 2000b, A\&A, 364, 937

Landi Degl'Innocenti, E. 1985, Sol. Phys., 99, 1

Manso Sainz, R., \& Landi Degl'Innocenti, E. 2002, A\&A, 394, 1093

Masnou-Seeuws, F., \& Roueff, E. 1972, Chem. Phys. L., 16, 593

Messiah, A. 1961, Mécanique Quantique (Paris: Dunod)

Murrell, J. N., \& Shaw, G. 1967, J. Chem. Phys., 46, 1768

Musher, J. I., \& Amos, A. T. 1967, Phys. Rev., 164, 31

Nienhuis, G. 1976, J. Phys. B: Atom. Molec. Phys., 9, 167

Omont, A. 1977, Prog. Quantum Electronics, 5, 69

Roueff, E. 1974, J. Phys. B, 7, 185

Sahal-Bréchot, S. 1977, ApJ, 213, 887

Sobelman, I. 1992, Atomic Spectra and Radiative Transitions (Heidelberg: Springer)

Unsöld, A. L. 1927, Z. Phys., 43, 574

Unsöld, A. L. 1955, Physik der Stern Atmosphären (Zweite Auflage) 